We thought to measure the value for patients we should let them participate in the project and asked them about what is of value for them and what are there goals. A good reason to start with a patient panel.

Objectives: For this reason we aimed to create a patient panel of rheumatology patients that are well informed and prepared to actively cooperate and participate in research, and co-design novel healthcare strategies.

Methods: Staff members (e.g. doctors and nurses) were asked to nominate patients that are expected to actively participate in the panel. Nominated patients were verbally approached and asked to participate. After mutual agreement a contract was signed were patients discretion was warranted, the capacity to handle confidential information was assessed and equality between members of the panel and staff was ensured. Enlargement of the panel is primarily staff-driven, but panel members are also invited to actively recruit other rheumatology patients. Results: The initial enrolment period lasted for six months. Thereafter, meetings were organized were discussion were held on various themes such as goals and value for rheumatology patients. Additionally, a focus group of rheumatoid arthritis (RA) patients was assembled to evaluate a PROMs measurement tool. To date, a number of four panel discussion have been held. Currently, our patient panel consists of 54 patients with all kinds of rheumatic diseases. Their demographic and clinical data are presented in Table 1

Table 1

\begin{tabular}{lc}
\hline & Value \\
\hline Patients (n) & 54 \\
Gender (\%male) & 17 \\
Age (yrs) & $51(20-77)$ \\
Disease duration (yrs) & $9(0-30)$ \\
Diagnosis (\%) & \\
- Rheumatoid Artrithis & 54 \\
- Arthrosis & 15 \\
- Fibromyalgia & 10 \\
- SpA (including APs and SA) & 12 \\
- Other & 9 \\
\hline
\end{tabular}

Our panel has been asked to participate in other (hospital-wide) programmes including the development and evaluation of a patient portal. We organize about three meetings for the whole panel every year and arrange focus group meetings to discuss specific subjects.

Conclusions: Panel members are very open and enthusiastic. Some quotes: "I'm happy to do something in return for the good care I receive.", and "I want to promote participation in scientific research". Deployment of patient participation for co-creating innovations alongside research is an asset these days to connect changes to patients perception.

In real live involving patient as an expert is not an effortless action for both patients and the expert care takers, it leads to satisfaction and an effective treatment.

Disclosure of Interest: None declared

DOI: 10.1136/annrheumdis-2017-eular.3454

\section{FRI0740-HPR ONLINE REMOTE MONITORING OF PATIENTS WITH RHEUMATOID ARTHRITIS: FIRST RESULTS OF A PILOT STUDY}

L. Renskers $^{1}$, S.A.A. Rongen ${ }^{1,2}$, S. Teerenstra ${ }^{3}$, M.E.J.L. Hulscher ${ }^{1}$,

P.L.C.M. van Riel ${ }^{1,2}$. ${ }^{1}$ IQ Healthcare, Radboud Institute for Health Sciences,

Radboud university medical center, Nijmegen; ${ }^{2}$ Rheumatology, Bernhoven,

Uden; ${ }^{3}$ Department for Health Evidence, Radboud Institute for Health Sciences, Radboud university medical center, Nijmegen, Netherlands

Background: Since the disease activity of patients with RA tends to fluctuate between visits and disease flares are easily missed during regular visits, patients might benefit from a more closely spaced determination of disease activity, which could be realised by iMonitor $^{1}$. iMonitor is a Software Medical Device developed and funded by Pfizer. This online system allows patients to complete three kinds of patient-reported outcome measures (PROMs): the Health Assessment Questionnaire (HAQ), Rheumatoid Arthritis Impact of Disease (RAID) and Rheumatoid Arthritis Disease Activity Index-5 (RADAI-5). iMonitor might contribute to identification of patients who need additional medical attention in between visits and reduction of visits for patients with stable disease activity. Objectives: To determine the degree to which the PROM-scores in iMonitor are associated with DAS28. Moreover, PROM preferences and completion rates were studied.

Methods: Patients were recruited at Bernhoven (Uden, the Netherlands) by an announcement on the hospital website, leaflets and meetings. Instruction classes were organised in which researchers assisted patients in using iMonitor. Patients indicated which $\operatorname{PROM}(\mathrm{s})$ they want to complete in iMonitor and chose reminder email frequency (weekly, two-, four, six, or eight-weekly). Descriptive analyses were used to describe characteristics of the study population. Scatter plots with regression equations were performed with DAS28 as dependent and PROM as independent variable to determine the association between DAS28 and PROMs. Moreover, Pearson's correlations were calculated. PROM-scores within the fourteen day window before and after DAS28 assessment were included. Results: In total 33 patients with RA were included, seventeen of them were female $(52 \%)$. Mean $( \pm S D)$ age was $56 \pm 11$ years. Seventeen patients $(52 \%)$ attended the instruction classes. Majority of patients $(n=10)$ chose all three PROMs to complete, nine patients chose RAID+RADAI-5, seven chose HAQ+RAID, three chose RAID, two chose RADAI-5 and two chose HAQ+RADAI-5. From March 2016 until December 2016, 435 RAID-values, 329 RADAl-5-values and 222 HAQ-values were gathered. When taking PROM-values within the fourteen day window before and after DAS28 assessment, 159 DAS28-values could be coupled to 320 PROM-values. Regression analysis showed the following proportions of explained variance $\left(R^{2}\right)$ : 0.17 for HAQ, 0.32 for RAID and 0.29 for RADAI-5. Pearson's correlation coefficients were 0.41 for $\mathrm{HAQ}, 0.57$ for RAID and 0.54 for RADAI-5. Most chosen reminder email frequency was four weeks ( $n=21)$. Completion rates (measured until December 31,2016 ) were $65 \%$ for patients with one week PROM-frequency and for patients with two, four, six and eight week frequency completion rates were $39 \%, 24 \%, 30 \%$ and $0 \%$, respectively.

Conclusions: RAID and RADAI- 5 were moderately associated with DAS28 and showed highest proportions of explained variance. The association between HAQ and DAS28 was weaker. Patients receiving a weekly reminder email showed highest completion rates. This pilot study is a first step towards personalised healthcare and patient involvement in online remote monitoring.

\section{References:}

[1] Medical and Educational Goods and Services (MEGS): iMonitor. 2014 Available from: http://www.pfizer.co.uk/content/medical-and-educational-goodsand-services-megs-imonitor.

Disclosure of Interest: L. Renskers Grant/research support from: Pfizer, S. Rongen: None declared, S. Teerenstra: None declared, M. Hulscher: None declared, P. van Riel Grant/research support from: Pfizer DOI: 10.1136/annrheumdis-2017-eular.5801

\section{FRI0741-HPR SCREENING FOR DEPRESSION AND ANXIETY IN AN OUTPATIENT RHEUMATOLOGY CLINIC USING VALIDATED SELF-APPLIED QUESTIONNAIRES}

L.F. Perez, A. Martinez, M. Moreno, L. Silveira. Reumatologia, Instituto Nacional de Cardiologia Ignacio Chavez, Mexico City, Mexico

Background: Anxiety and depression are often present in chronic rheumatic diseases. Recognition of these psychological disorders is fundamental for proper patient management. The absence of screening leaves more than $>50 \%$ of patients with depression unidentified. Patient Help Questionnaire-9 (PHQ-9) and General Anxiety Disorder-7 (GAD-7) are two validated self-applied questionnaires that are appropriate to assess the presence of, depression and anxiety, respectively.

Objectives: 1) To assess the prevalence of depression and anxiety in a hospital based outpatient Rheumatology clinic and 2) To provide the attending physician with appropriate instruments that allow a rapid orientation on the psychological status of her/his patient.

Methods: Consecutive patients that attended our outpatient Rheumatology clinic from March to June 2016 were invited to participate in this crosssectional study. Participants filled out PHQ-9 and GAD-7 in the waiting room. The prevalence and severity of anxiety and depression were calculated for the most prevalent diagnoses.

Results: A total of 410 patients were recruited; 339 (82.8\%) were female. Overall, $191(46.6 \%)$ patients reported depressive symptoms (PHQ-9>5). Of them, 87 $(21.2 \%)$ were classified as having moderate depression or higher (PHQ-9 $>10)$. Prevalence of depression and anxiety among study participants according to each rheumatic disease is depicted in Table 1. Prevalence of moderate or severe depression was significantly different among various rheumatic diseases $(p=0.001)$. Regarding anxiety symptoms, they were reported in $168(40.7 \%) ; 67$ $(16.2 \%)$ of them had moderate or severe anxiety.

\begin{tabular}{|l|l|l|l|}
\hline Disease & N & $\begin{array}{l}\text { Moderate or severe } \\
\text { depression }\end{array}$ & $\begin{array}{l}\text { Moderate or } \\
\text { severe anxiety }\end{array}$ \\
\hline SLE & $99(24.1 \%)$ & $16(16.2 \%)$ & $12(12.1 \%)$ \\
\hline RA & $107(26.1 \%)$ & $26(24.3 \%)$ & $17(15.9 \%)$ \\
\hline FM & $87(21.3 \%)$ & $\begin{array}{l}30(34.5 \%) \\
p=0.001\end{array}$ & $\begin{array}{l}27(31 \%) \\
p=0.001\end{array}$ \\
\hline OA & $69(16.8 \%)$ & $\begin{array}{l}25(36.2 \%) \\
p=0.001\end{array}$ & $\begin{array}{l}22(31.9 \%) \\
p=0.001\end{array}$ \\
\hline SSC & $18(4.4 \%)$ & $13(26 \%)$ & $8(16 \%)$ \\
\hline $\begin{array}{l}\text { Inflammatory } \\
\text { myopathies }\end{array}$ & $14(3.4 \%)$ & $4(28.6 \%)$ & $2(14.3 \%)$ \\
\hline Osteoporosis & $49(12 \%)$ & $14(28.6 \%)$ & $9(18.4 \%)$ \\
\hline APS & $42(10.2 \%)$ & $\begin{array}{l}1(2.4 \%) \\
p=0.001\end{array}$ & $3(7.1 \%)$ \\
\hline
\end{tabular}

Table 1. Prevalence of depression and anxiety amone study participants according to each rheumatic disease. (SLE - Systemic lupus erythematosus, RA - Rheumatoid Arthritis, FM Fibromyalgia, OA - Osteoarthritis, SSC-Systemic sclerosis, APS - Antiphospholipid syndrome.

Conclusions: This cross-sectional study shows that anxiety and depression are frequent in the Rheumatology clinic. We demonstrated that the use of a self-applied screening tool can help clinicians to properly detect depression and anxiety associated with diverse rheumatic diseases. Specia attention should be paid to patients with fibromyalgia and osteoarthritis. 
Disclosure of Interest: None declared

DOI: 10.1136/annrheumdis-2017-eular.6765

\section{FRI0742-HPR DOES THE USE OF TECHNOLOGICAL DEVICES IMPROVE THE RELIABILITY OF MEASURING THE ACTIVE CERVICAL RANGE OF MOTION IN PATIENTS WITH NECK PAIN? A SYSTEMATIC REVIEW WITH META-REGRESSION}

A. Rondoni ${ }^{1}$, G. Rossettini ${ }^{1}$, M. Strobe ${ }^{1}$, F. Gallo ${ }^{2}$, D. Ristori ${ }^{1}$, M. Testa $^{1}{ }^{1}$ ${ }^{1}$ Department of Neuroscience, Rehabilitation, Ophthalmology, Genetics, Maternal and Child Health, University of Genova, Savona; ${ }^{2}$ Department of Health Sciences, University of Genova, Genova, Italy

Background: Economic impact of neck pain shows an increasing trend. In order to limit the costs of spine disorders management, it is important to assess clinical efficacy and the cost-worthiness of new technological devices, recently introduced in physical therapist's clinical practice

Objectives: This systematic review compares, in patients with non-specific neck pain, the reliability of measures of Active Cervical Range of Motion (ACROM) detected with technological devices with those assessed with low cost, commonuse devices. As secondary outcomes, it was investigated if ACROM reliability depends on the plane on which the measured movement is performed

Methods: The literature search was carried out in Medline, Scopus, Embase, The Cochrane Library, CINHAL, PEDro and grey literature until August 2016. Inclusion criteria were: reliability design, population of adults with nonspecific neck pain, examiners of any level of experience, measures repeated at least twice and statistics indexes on reliability. Exclusion criteria were: other study designs, asymptomatic population or mixed population, single or none ACROM measure, inadequate statistics. The risk of bias was assessed by QAREL. It was considered inexpensive a device that costs at maximum 500 euros. A Univariate, and a Multivariate Analysis, were performed by using the Linear Mixed-Effect Model Results: Searching the databases yielded 35,151 records. Nine studies met all eligibility criteria. The QAREL mean score of the selected studies was 3.7 out of 11. No significant effect of the type of device (inexpensive versus expensive) on ICC was observed for intra-rater (ICC $=0.93 v s 0.91 \mathrm{p}$-value $>0.99$ ) and inter-rater reliability $($ ICC $=0.80 \mathrm{vs} 0.87 \mathrm{p}$-value $>0.99)$ [Table 1]. The plane of movement did not affect inter-rater reliability ( $p$-value $=0.11$ ) while significantly influenced the intra-rater reliability $(p$-value $=0.0001)$ assessed with low-cost devices. Intra-rater reliability significantly decreases $(p$-value $=0.0129)$ in frontal plane movements (side bending) compared with movement on the sagittal plane (flexion-extension).

Table 1. Comparison of intra and inter-rater reliability between tool types

\begin{tabular}{lccc}
\hline Characteristics & ICC Descriptive statistics & \multicolumn{2}{c}{ Mixed effect model } \\
\cline { 3 - 4 } & Mean (SD) & $\beta(95 \% \mathrm{Cl})$ & $\mathrm{p}$-value \\
\hline $\begin{array}{l}\text { Intra-rater reliability } \\
\text { Tool Type }\end{array}$ & & 0.99 \\
$\quad$ Expensive & $0.91(0.07)$ & $0.01(-0.04: 0.06)$ & \\
$\quad$ Inexpensive & $0.93(0.02)$ & 0 & 0.56 \\
$\quad$ Direction & & $0.03(-0.01: 0.07)$ & \\
$\quad$ Flexion \& Extension & $0.91(0.07)$ & $0.01(-0.02: 0.04)$ & \\
$\quad$ Rotation & $0.95(0.02)$ & $0.00(-0.01: 0.01)$ & 0.79 \\
$\quad$ Side Bending & $0.92(0.01)$ & & 0.99 \\
Age & rho $=0.29$ & 0 & \\
Inter-rater reliability & & $0.03(-0.15: 0.21)$ & \\
Tool Type & & 0 & 0.06 \\
$\quad$ Expensive & $0.87(0.09)$ & $0.04(-0.02: 0.11)$ & \\
$\quad$ Inexpensive & $0.80(0.12)$ & $-0.04(-0.10: 0.01)$ & \\
Direction & & & \\
$\quad$ Flexion \& Extension & $0.82(0.09)$ & $0.01(-0.01: 0.03)$ & 0.43 \\
$\quad$ Rotation & $0.86(0.11)$ & & \\
$\quad$ Side Bending & $0.77(0.13)$ & & \\
Age & rho $=0.58$ & & \\
\hline
\end{tabular}

Conclusions: The use of expensive devices to measure ACROM in adults with nonspecific neck pain does not seem to improve the reliability of the assessment. The assessment of side bending showed the lowest level of inter-raters reliability. Since the quality of the analysed studies is low, the conclusion of the present study should be taken cautiously

Disclosure of Interest: None declared

DOI: 10.1136/annrheumdis-2017-eular.3991

\section{FRI0743-HPR THE ASSOCIATION OF PHYSICAL FITNESS COMPONENTS WITH SLEEP QUALITY IN WOMEN WITH FIBROMYALGIA: THE AL-ÁNDALUS PROJECT}

M. Borges-Cosic ${ }^{1}$, V. Segura-Jiménez ${ }^{2}$, F. Estévez-López ${ }^{1,3}$,

I.C. Álvarez-Gallardo ${ }^{2}$, V.A. Aparicio ${ }^{4}$, P. Acosta-Manzano ${ }^{1}$,

B. Gavilán-Carrera ${ }^{1}$, M. Delgado-Fernández ${ }^{1}$. ${ }^{1}$ Physical education and sport,

University of Granada, Granada; ${ }^{2}$ Physical Education, University of Cádiz, Cádiz,

Spain; ${ }^{3}$ Department of Psychology, University of Utrecht, Utrecht, Netherlands;

${ }^{4}$ Department of Physiology, University of Granada, Granada, Spain

Background: Fibromyalgia women report poorer sleep quality, fewer hours of sleep, greater nighttime awakenings, and non-restorative sleep compared with healthy people and other clinical populations (Diaz-Piedra et al., 2015). Previous literature has shown a positive effect of exercise training on sleep quality in women with fibromyalgia (Munguía-Izquierdo et al., 2008). Given the close relationship between exercising and physical fitness improvement, it is important to ascertain the individual influence of physical fitness components on sleep quality in this population.

Objectives: To examine the individual association of physical fitness components with sleep quality in women with fibromyalgia.

Methods: A total of 444 women with fibromyalgia (means $\pm s d=51.3 \pm 7.5$ years old) from Andalusia (southern Spain) were included in this cross-sectional study. The Senior Fitness test battery was used to assess physical fitness. This battery consists of the chair sit-and-reach, back scratch, 30-second chair stand, arm curl, 8-foot up-and-go (lower score indicates better performance) and 6-minute walk tests, which assess lower body flexibility, upper body flexibility, lower body strength, upper body strength, speed agility and cardiorespiratory fitness, respectively. Sleep quality was measured using the total score of the Pittsburgh Sleep Quality Index (PSQI) questionnaire (greater score indicates worse sleep quality). Linear regression was used to explore the association of each physical fitness component with global sleep quality. Analyses were controlled for age, total body fat percentage, marital status, educational level, medication for relaxation/sleep and regular menstruation.

Results: Lower body flexibility $(b=-0.033, \beta=-0.103)$, upper body flexibility $(b=-$ $0.031, \beta=-0.096)$, lower body strength $(b=-0.191, \beta=-0.157)$, upper body strength $(b=-0.111, \beta=-0.142)$ and cardiorespiratory fitness $(b=-0.008, \beta=-0.154)$ were inversely associated with greater scores in the PSQI questionnaire (all, $p<0.05$ ). Speed-agility $(b=0.282, \beta=0.128)$ was positively associated with the score in the PSQI $(p<0.01)$

Conclusions: The current study showed that higher physical fitness is generally associated with better sleep quality in women with fibromyalgia. It is important to mention that all the physical fitness components seem to be associated with sleep quality. Lower body strength and aerobic capacity were the components that showed the strongest associations with sleep quality. Future studies are warranted to elucidate the causality of these relationships.

References:

[1] Diaz-Piedra, Carolina et al. 2015. Sleep Disturbances of Adult Women Suffering from Fibromyalgia: Asystematic Review of Observational Studies. Sleep Medicine Reviews 21:86-99.

[2] Munguía-Izquierdo, D. \& Legaz-Arrese, A. Assessment of the effects of aquatic therapy on global symptomatology in patients with fibromialgia síndrome: a randomized controlled trial. Arch. Phys. Med. Rehabil. 2008, 89:2250-2257.

Acknowledgements: This study was supported by the Spanish Ministry of Economy and Competitiveness (I+D+I DEP2010-15639; DEP2013-40908-R).

Disclosure of Interest: None declared

DOI: 10.1136/annrheumdis-2017-eular.4801

\section{FRI0744-HPR THE EFFECTIVENESS OF THE LOWER DOSE OF LASER TREATMENT ON KNEE OSTEOARTHRITIS}

E. Ekiz ${ }^{1}$, K. Capaci ${ }^{2}$, M. Korkmaz ${ }^{3} .{ }^{1}$ Physical therapy and rehabilitation, Alaşehir State Hospital, Manisa; ${ }^{2}$ Physical therapy and rehabilitation, Ege University, Izmir; ${ }^{3}$ Physical therapy and rehabilitation, Afyonkarahisar State Hospital, Afyonkarahisar, Turkey

Background: During knee osteoarthritis (OA) therapy; medications, physical therapy modalities, exercises and surgical procedures are used. In previous studies, the benefits of low level laser therapy (LLLT) for pain in the low back, shoulder, elbow, and hand have reported.

Objectives: In this study we aimed to investigate the effectiveness of LLLT on pain, functional status, and quality of life in patients with knee osteoarthritis.

Methods: Patients with Kellgren-Lawrence stage 2-3 knee osteoarthritis were enrolled. It was planned as a prospective, randomized and double-blind study. Group 1 received active laser therapy and group 2 placebo laser therapy. Patients have been provided active/placebo laser therapy for two weeks ( 5 days a week, a total of 10 sessions). Patients were evaluated before, immediately after and 1 or 3 months after treatment. Outcome measurements included pain intensity at rest and at movement on visual analog scale, knee function using Western Ontario McMaster Universities Osteoarthritis Index (WOMAC) scale, active/passive joint range of movement, quality of life using Short form 36 (SF-36), and 15-meter walking distance, painless walking distance.

Results: In this study, we observed improvements in knee pain, 15-meter walking distance, WOMAC pain, stiffness and function scores, Lequesne index, and SF-36 physical function and social function $(p<0.05)$ in active LLLT group. Also, significant improvements were detected in SF-36 physical role, mental health, vitality and emotional role parameters compared with placebo laser group $(p>0.05)$.

Conclusions: The present study demonstrated that LLLT is safe and effective upon pain and functional parameters on knee osteoarthritis. However, we think further long-term studies which will include more patients in order to determine the priority and effectiviness are required.

Disclosure of Interest: None declared

DOI: 10.1136/annrheumdis-2017-eular.5187 